Louisiana State University

LSU Digital Commons

$11-1-2004$

\title{
Glycine N-methyltransferases: A comparison of the crystal structures and kinetic properties of recombinant human, mouse and rat enzymes
}

\author{
Svetlana Pakhomova \\ Louisiana State University \\ Zigmund Luka \\ Vanderbilt University School of Medicine \\ Steffi Grohmann \\ Louisiana State University \\ Conrad Wagner \\ Vanderbilt University School of Medicine \\ Marcia E. Newcomer \\ Louisiana State University
}

Follow this and additional works at: https://digitalcommons.Isu.edu/biosci_pubs

\section{Recommended Citation}

Pakhomova, S., Luka, Z., Grohmann, S., Wagner, C., \& Newcomer, M. (2004). Glycine N-methyltransferases: A comparison of the crystal structures and kinetic properties of recombinant human, mouse and rat enzymes. Proteins: Structure, Function and Genetics, 57 (2), 331-337. https://doi.org/10.1002/prot.20209 


\title{
Glycine N-Methyltransferases: A Comparison of the Crystal Structures and Kinetic Properties of Recombinant Human, Mouse and Rat Enzymes
}

\author{
Svetlana Pakhomova, ${ }^{1}$ Zigmund Luka, ${ }^{2}$ Steffi Grohmann, ${ }^{1}$ Conrad Wagner, ${ }^{2 *}$ and Marcia E. Newcomer ${ }^{1}$ \\ ${ }^{1}$ Department of Biological Sciences, Louisiana State University, Baton Rouge, Louisiana \\ ${ }^{2}$ Vanderbilt University School of Medicine, Department of Biochemistry, Nashville, Tennessee
}

\begin{abstract}
Glycine N-methyltransferases (GNMTs) from three mammalian sources were compared with respect to their crystal structures and kinetic parameters. The crystal structure for the rat enzyme was published previously. Human and mouse GNMT were expressed in Escherichia coli in order to determine their crystal structures. Mouse GNMT was crystallized in two crystal forms, a monoclinic form and a tetragonal form. Comparison of the three structures reveals subtle differences, which may relate to the different kinetic properties of the enzymes.

The flexible character of several loops surrounding the active site, along with an analysis of the active site boundaries, indicates that the observed conformations of human and mouse GNMTs are more open than that of the rat enzyme. There is an increase in $\boldsymbol{k}_{\text {cat }}$ when going from rat to mouse to human, suggesting a correlation with the increased flexibility of some structural elements of the respective enzymes. Proteins 2004;57:331-337.

๑ 2004 Wiley-Liss, Inc.
\end{abstract}

Key words: glycine N-methyltransferase; X-ray crystallography; conformations; kinetics

\section{INTRODUCTION}

The enzyme glycine N-methyltransferase (GNMT) is abundant in mammalian liver, comprising between 0.5 and $3 \%$ of the cytosolic protein, ${ }^{1-3}$ depending on the species. Its relative abundance may indicate an important physiological role for its activity. GNMT catalyzes the transfer of a methyl group from S-adenosylmethionine (SAM) to glycine to yield S-adenosylhomocysteine (SAH) and N-methylglycine (sarcosine). The enzyme appears to play a significant role in maintaining the physiologically appropriate SAM-to-SAH ratio, which is thought to be a critical component of the regulation of the methylation potential of the cell. ${ }^{4}$ In addition, GNMT is bound to 5-methyl-tetrahydrofolate pentaglutamate in vivo, ${ }^{5}$ and the folate coenzyme behaves as an inhibitor of GNMT in the enzymatic reaction. ${ }^{6}$ This is part of a regulatory scheme that ties the de novo synthesis of methyl groups by the one-carbon folate pool to the availability of pre-formed methyl groups in methionine. ${ }^{7}$ GNMT is abundant in the liver, the pancreas and apparently also in the prostate. It is also found in cells that carry out secretion. ${ }^{8}$ The protein sequence of GNMT from cloned cDNAs of several organisms, including humans, show a high level of sequence identity $(90 \%$ of the amino acids are identical in the human, rat and mouse forms). ${ }^{3}$ All GNMTs that have been purified are tetramers consisting of four identical monomers, each having a $M_{r}$ of $32.5 \mathrm{kDa}$. All GNMTs bear an $\mathrm{N}$-terminal acetyl group on the initial valine, ${ }^{3}$ and it has been shown that in the rat liver enzyme the enzyme is phosphorylated. ${ }^{9}$

Detailed structural and kinetic studies of the rat recombinant form of the enzyme have been reported, and a mechanism for the enzymatic reaction has been proposed based on crystal structures of rat GNMT. ${ }^{10-13}$ According to the proposed mechanism, significant conformational changes occur during the methyltransferase reaction. As defined by Fu et al., ${ }^{10}$ the rat GNMT monomer is compoised of three domains: the $\mathrm{N}$-terminal domain (residues 1-36), the C-terminal domain (residues 37-175 and 243292) and the S-domain (residues 176-242). Three basic conformational states of rat GNMT, an 'inactive' or 'closed' structure, an 'open' structure and an 'active' structure have been described..$^{10-13}$

In the 'closed' conformation (observed in the GNMT-SAMacetate structure ${ }^{10}$ and the apo-form ${ }^{11}$ ), the active sites of GNMT in each monomer are covered by the N-terminal domain from the adjacent subunit. No apparent entrance to or exit from the active site is observed. This conformational state was proposed to be the 'initial stage' of the methyltransferase reaction.

In the 'open' conformation (R175K GNMT-SAH structure ${ }^{12}$ ), the entire $\mathrm{N}$-terminal domain is highly disordered, and the S-domain is significantly shifted relative to its orientation in the closed conformation. It has been suggested that the $\mathrm{N}$-terminal domain must move out from the active site of the adjacent subunit into the solvent region in order to provide an entrance for SAM and glycine into the active site. ${ }^{12}$ The SAH binding site in this structure corresponds to the SAM binding site in other

S. Pakhomova and Z. Luka contributed equally to this work.

*Correspondence to: Conrad Wagner, Department of Biochemistry, Vanderbilt University School of Medicine, 620 Light Hall, Nashville, TN 37232. E-mail:conrad.wagner@vanderbilt.edu.

Received 20 November 2003; Revised 2 April 2004, 19 April 2004; Accepted 27 April 2004

Published online 22 June 2004 in Wiley InterScience (www.interscience.wiley.com). DOI: 10.1002/prot.20209 
methyltransferases. This conformational state has been linked to the product release stage in the latest work by the same authors. ${ }^{13}$

In the 'active' conformation (R175K GNMT-SAM structure $\left.{ }^{13}\right)$, described as near the transition state or active conformation, the $\mathrm{N}$-terminus is directed into the solvent. However, in contrast to what is described as the 'open' form of the enzyme, only residues $1-18$ of the $\mathrm{N}$-terminus are highly disordered. In addition, residues 126-127 and 226-234 are slightly disordered. In this structure, the SAM binding site is consistent with the SAM binding site in other methyltransferases.

In order to determine whether the proposed mechanism for the rat enzyme is consistent with the structures and kinetic parameters of other mammalian GNMT enzymes, crystallographic and kinetic studies were undertaken with human and mouse forms of the enzyme.

A comparison of the properties of GNMTs from human, rat, rabbit and pig livers was carried out by Ogawa and coworkers. ${ }^{14}$ The enzymes were only partially purified. Nevertheless, the values for the substrate concentrations at which half maximal activity $\left([S]_{0.5}\right)$ for SAM and glycine was reached of the enzyme from the rat were much lower (by 18 to $5 \%$ ) than the corresponding values from the human enzyme. The $[S]_{0.5}$ values for SAM and glycine for the rat enzyme were also much lower than the corresponding values for the rabbit and pig enzymes.

The identification of naturally occurring mutations in the enzyme necessitated a structural determination of the human form of GNMT. We discovered two sets of patients with mutations in the coding region of the gene for GNMT. In one family, both brother and sister were found to be compound heterozygotes with an L49P mutation on one allele and $\mathrm{H} 176 \mathrm{~N}$ on the other. ${ }^{15,16}$ The other patient had an N140S mutation. ${ }^{17}$ In each case, when the L49P and the $\mathrm{H} 176 \mathrm{~N}$ mutations were expressed in $E$. coli, the recombinant enzyme displayed greatly reduced activity. ${ }^{18}$ When the N140S mutation was expressed in E. coli, it had almost no activity. Although the structure of the rat enzyme can help to interpret the consequences of these mutations, a model for the human enzyme would reveal any unforeseen structural differences between the native rat and human enzymes. We report here the structures of the human and mouse enzymes and provide a structural interpretation for differences we observed in their kinetic parameters.

\section{MATERIALS AND METHODS Protein Preparation}

GNMT cDNAs were cloned and expressed in the pET$17 \mathrm{~b}$ expression vector, as reported earlier. ${ }^{19}$ Briefly, the GNMT cDNAs were cloned from the mouse and human poly-A+ RNA and from the rat Marathon-Ready RNA (Clontech, Palo Alto, CA). Cloned cDNAs were introduced into pET-17b expression vector. Expression was completed according to the standard approach for that expression system at $20^{\circ} \mathrm{C}$ for $17-18 \mathrm{~h}$ with $1 \mathrm{~m} M$ IPTG.

GNMT was isolated and purified by ammonium sulfate precipitation of the enzyme from crude extract, followed by ion-exchange chromatography on a DE-52 column, as described previously. ${ }^{19}$ Further purification was performed with a Sephacryl S-200 size-exclusion column. The final purity of all GNMT samples was at least 95-97\%.

\section{Protein Crystallization Human GNMT}

Crystals of human GNMT were grown at $4^{\circ} \mathrm{C}$ by the hanging drop vapor diffusion method after mixing $1 \mu \mathrm{L}$ of the enzyme at $8.4 \mathrm{mg} / \mathrm{mL}$ in $50 \mathrm{~m} M$ Tris- $\mathrm{HCl} \mathrm{pH} 8.0,200$ $\mathrm{m} M \mathrm{NaCl}, 14 \mathrm{~m} M \beta$-mercaptoethanol, $1 \mathrm{~m} M$ EDTA with 1 $\mu \mathrm{L}$ of the reservoir solution $[9-11 \%(\mathrm{w} / \mathrm{v})$ polyethylene glycol 4000, 0.1M Na-citrate pH 5.6-6.0]. Crystals usually appeared overnight and grew to full size within 1-2 days.

\section{Mouse GNMT}

Crystals of mouse GNMT were grown at $22^{\circ} \mathrm{C}$ by the hanging drop vapor diffusion method by mixing equal volumes of enzyme at $5.7 \mathrm{mg} / \mathrm{mL}$ in $20 \mathrm{mM}$ Tris- $\mathrm{HCl} \mathrm{pH}$ 7.5, $90 \mathrm{~m} M \mathrm{NaCl}, 14 \mathrm{~m} M \beta$-mercaptoethanol, $1 \mathrm{~m} M$ EDTA and the reservoir solution $[15 \%(\mathrm{w} / \mathrm{v})$ polyethylene glycol 4000, 0.1M Tris-HCl pH 8.5 and 5\% isopropanol]. Crystals of both forms $\left(\mathrm{P} 2_{1}\right.$ and $\left.\mathrm{P} 4_{1} 2_{1} 2\right)$ grew under the same conditions and often in the same drop.

\section{Data Collection}

Prior to data collection, a suitable crystal was dipped for $30 \mathrm{~s}$ in a modified mother solution with the addition of $20 \%$ ethylene glycol as a cryo-protectant. All diffraction data were collected at $100 \mathrm{~K}$ with a cryo-stream cooler from Oxford Cryojet with a $345 \mathrm{~mm}$ MAR Research imaging plate detector mounted on a NONIUS FR591 rotating anode generator $\left(\mathrm{CuK}_{\alpha}\right.$ radiation). Data were processed with DENZO ${ }^{20}$ or Mosflm ${ }^{21}$ and scaled using Scalepack ${ }^{20}$ or Scala. ${ }^{22}$ Data collection and data processing statistics are given in Table I.

\section{Crystal Structure Determination}

All crystal structures were solved by the molecular replacement $(\mathrm{MR})$ procedure implemented in the CNS package. ${ }^{23}$ A dimer of the rat GNMT structure [Protein Data Bank (PDB) accession code 1XVA] was used as a search model for human GNMT and the tetragonal form of mouse GNMT; the structure of the monoclinic form of mouse GNMT was solved using a dimer from the tetragonal form of the same protein. The positioned MR models were refined using the maximum likelihood refinement in REFMAC. ${ }^{22}$ Twofold non-crystallographic symmetry restraints and bulk solvent corrections were applied in every case. The program $\mathrm{O}^{24}$ was used to build the initial models and throughout the refinement. Refinement statistics are listed in Table I. Details of refinement of each of the structures follow.

\section{Human GNMT}

No significant electron density was observed for residues 1-4, 126-128, 225-235 of monomer A or 1-4, 226-235 of monomer $\mathrm{B}$, an indication that these regions are highly mobile or disordered. An electron density in the $F_{o}-F_{c}$ map, which can be attributed to a citrate molecule, was found in the active site. Additional electron densities on 
TABLE I. Data Collection and Refinement Statistics

\begin{tabular}{|c|c|c|c|}
\hline & Human GNMT & $\begin{array}{c}\text { Mouse GNMT } \\
\text { (tetr. form) }\end{array}$ & $\begin{array}{l}\text { Mouse GNMT } \\
\text { (mon. form) }\end{array}$ \\
\hline Wavelength & 1.5418 & 1.5418 & 1.5418 \\
\hline Resolution $(\AA)$ & 2.55 & 2.95 & 3.05 \\
\hline Space group & $P 2_{1} 2_{1} 2$ & $P 4_{1} 2_{1} 2$ & $P 2_{1}$ \\
\hline \multicolumn{4}{|l|}{ Cell dimensions } \\
\hline$a(\AA)$ & 75.84 & 70.70 & 107.98 \\
\hline$b(\AA)$ & 83.23 & 70.70 & 108.30 \\
\hline$c(\AA)$ & 114.88 & 266.03 & 119.01 \\
\hline$\alpha\left({ }^{\circ}\right)$ & 90.00 & 90.00 & 90.00 \\
\hline$\beta\left(^{\circ}\right)$ & 90.00 & 90.00 & 93.66 \\
\hline$\gamma\left({ }^{\circ}\right)$ & 90.00 & 90.00 & 90.00 \\
\hline Number of monomers per asymmetric unit & 2 & 2 & 8 \\
\hline Number of unique reflections & 23864 & 14973 & 51310 \\
\hline$R_{\text {sym }}^{\text {ab }(\%)}$ & $5.1(38.9)$ & $6.8(43.1)$ & $6.6(40.3)$ \\
\hline Completeness (\%) & $98.3(98.3)$ & $99.7(99.7)$ & $99.2(100)$ \\
\hline Redundancies & 3.7 & 3.7 & 3.6 \\
\hline$I / \sigma(I)$ & $9.9(1.8)$ & $6.7(1.8)$ & $19.6(3.1)$ \\
\hline \multicolumn{4}{|l|}{ Refinement Statistics } \\
\hline Resolution range & $12-2.55$ & $10.5-2.95$ & $13-3.05$ \\
\hline Number of reflections used in refinement & 19506 & 13784 & 46863 \\
\hline$\sigma$ Cutoff used in refinement & none & none & none \\
\hline$R / R_{\text {free }}{ }^{\mathrm{c}}(\%)$ & $22.18 / 26.61$ & $20.68 / 27.27$ & $22.22 / 28.51$ \\
\hline \multicolumn{4}{|l|}{ Number of refined atoms } \\
\hline Protein & 4230 & 4356 & 17552 \\
\hline Heterogen atoms & 42 & 32 & 40 \\
\hline Water & 60 & 21 & 14 \\
\hline Average B-factors $\left(\AA^{2}\right)$ & 33.7 & 27.2 & 24.7 \\
\hline \multicolumn{4}{|l|}{ RMS deviations } \\
\hline Bonds $(\AA)$ & 0.027 & 0.024 & 0.017 \\
\hline Angles $(\AA)$ & 2.167 & 2.167 & 1.650 \\
\hline \multicolumn{4}{|l|}{ Ramachandran statistics } \\
\hline Most favored regions (\%) & 88.6 & 83.9 & 86.1 \\
\hline Disallowed regions $(\%)$ & 0.0 & 0.0 & 0.0 \\
\hline
\end{tabular}

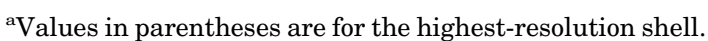

${ }^{\mathrm{b}} R_{\mathrm{sym}}=\Sigma\left|I_{i}-<I>\right| / \Sigma I_{i}$, where $I_{i}$ is the intensity of the $i$ in observation and $\langle I>$ is the mean intensity of the reflection

${ }^{c} R=\Sigma|| F_{o}|-| F_{c}|| \Sigma\left|F_{o}\right|$, where $F_{o}$ and $F_{c}$ are the observed and calculated structure factor amplitudes. $R_{\text {free }}$ is calculated using $6.9 \%, 5.6 \%$ and $3.0 \%$ of reflections omitted from the refinement for the human GNMT, tetragonal form and monoclinic form of mouse GNMT respectively.

cysteines 187 (monomer A) and 187, 264 and 284 (monomer B) were interpreted as coming from covalently-bound $\beta$-mercaptoethanol (BME) molecules, as the protein buffer contained BME.

\section{Tetragonal Form of Mouse GNMT}

The final model consisted of residues 1-224 and 234292 for monomer A and 1-225 and 234-292 for monomer B. No electron density was observed for residues A225A233 or B226-B233. A difference Fourier map suggests the presence of a Tris molecule in the active site of the protein. Covalently bound BME was modeled at cysteines A146, A246, B146 and B262.

\section{Monoclinic Form of Mouse GNMT}

In this crystal form, an asymmetric unit is composed of two tetramers. There is no electron density for residues A224-A234, B225-B234, C228-C234, D226-D231, E228E233, F225-F233, G225-G233 and H225-H234 (A-H refer to different monomers). The active site does not have any 'extra' electron density such as that attributed to a Tris molecule in the tetragonal form. Covalently-bound BME residues were found for cysteines A262, A282, B57, D185, D246, D262, E246, F282, G246 and G282.

The refined coordinates have been deposited in the PDB with the following accession codes: human GNMT, 1R74; tetragonal form of mouse GNMT, 1R8X; monoclinic form of mouse GNMT, 1R8Y.

\section{GNMT Activity}

GNMT activity was assayed using the charcoal adsorption method, as described earlier. ${ }^{25}$ A $100 \mu \mathrm{L}$ reaction mixture contained $200 \mathrm{~m} M$ Tris-HCl buffer $\mathrm{pH}$ 7.5, $5 \mathrm{mM}$ dithiotreitol (DTT), about $2 \mu \mathrm{g}$ enzyme and both substrates, SAM and glycine, at the appropriate concentrations. Each incubation also contained $\left[{ }^{3} \mathrm{H}-\mathrm{CH}_{3}\right]-\mathrm{SAM}(3-$ $5 \times 10^{3} \mathrm{dpm}$ ). After incubation at $25^{\circ} \mathrm{C}$ for $15 \mathrm{~min}$, the reaction was stopped by the addition of $50 \mu \mathrm{L} 10 \%$ trichloroacetic acid. A $250 \mu \mathrm{L}$ suspension of acid-treated charcoal in $0.1 M$ acetic acid was added, and the suspension 


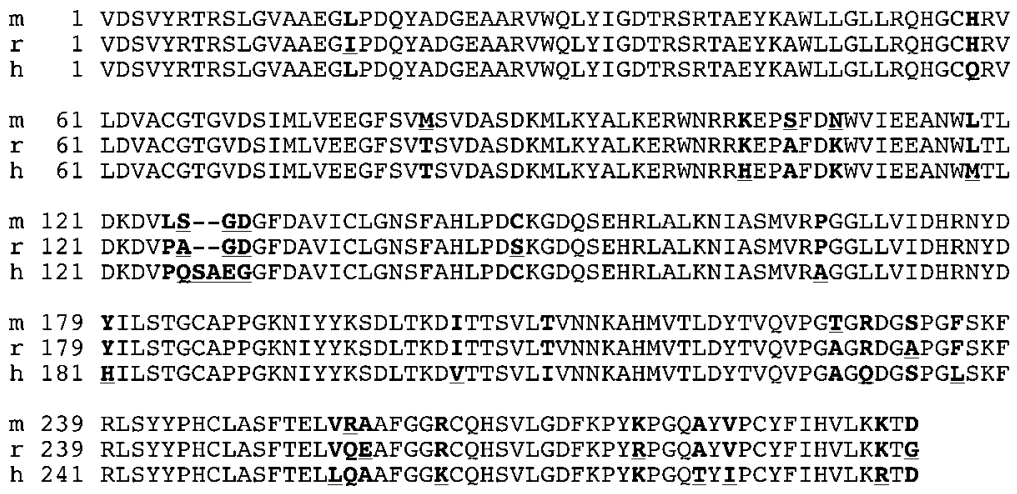

Fig. 1. Sequence alignment of mouse ( $m)$, rat ( $r$ ) and human (h) GNMTs.

TABLE II. Kinetic Parameters of Human, Mouse and Rat GNMTs.

\begin{tabular}{lccccc}
\hline Protein & $k_{\text {cat }}\left(\min ^{-1}\right)$ & $K_{m}(\mathrm{SAM})(\mu M)$ & $\begin{array}{c}k_{c a t} / K_{m}(\mathrm{SAM}) \\
\left(\min ^{-1} / \mu M\right)\end{array}$ & $K_{m}(\mathrm{gly})(\mathrm{m} M)$ & $\begin{array}{c}k_{c a t} / K_{m}(\mathrm{gly}) \\
\left(\mathrm{min}^{-1} / \mathrm{m} M\right)\end{array}$ \\
\hline Human* & 96.4 & 281.0 & 0.34 & 12.2 & 7.90 \\
Mouse & 43.6 & 180.3 & 0.24 & 3.6 & 12.11 \\
Rat & 33.5 & 107.0 & 0.31 & 2.0 & 16.75 \\
\hline
\end{tabular}

Data from (ref 18).

was incubated on ice for $15 \mathrm{~min}$. After centrifugation in a microcentrifuge, the radioactivity in the supernatant was counted with a Tri-Carb Liquid Scintillation Analyzer (Packard Instrument Co., Meriden, CT). $K_{m}$ values for SAM and glycine were obtained by varying the concentration of one substrate in the presence of saturating concentrations of the other.

\section{Quaternary Structure}

The quaternary structures of the expressed proteins were determined by size-exclusion chromatography on the ÄKTA Purifier System (Amersham Pharmacia, Piscataway, NJ). Chromatography of the native proteins was performed on a Superose-12 column in a buffer composed of $50 \mathrm{~m} M$ Tris- $\mathrm{HCl} \mathrm{pH} \mathrm{7.5,} 150 \mathrm{mM} \mathrm{NaCl}, 14 \mathrm{~m} M$ BME at flow rate of $0.5 \mathrm{~mL} / \mathrm{min}$ at $22^{\circ} \mathrm{C}$. The sample volume was $100 \mu \mathrm{L}$, and the column was calibrated with chymotrypsin, ovalbumin, bovine serum albumin and aldolase.

\section{Protein Assay}

The concentrations of protein samples were determined by the BCA method using bovine serum albumin as a standard. ${ }^{26}$ Protein purity was determined by SDS-PAGE electrophoresis and by N-terminal Edman sequencing. Gels were documented using a NucleoVision 760 Imaging Workstation (NucleoTech, San Mateo, CA).

\section{RESULTS AND DISCUSSION}

The primary goal of our work was to study how differences in the amino acid sequences of three glycine $\mathrm{N}$ methyltransferases could affect the catalytic properties of these enzymes. The amino acid sequences of rat, mouse and human GNMTs show approximately 90\% sequence identity (Fig. 1). The rat and mouse proteins consist of 292 amino acid residues, while the human GNMT consists of
294 residues due to an insertion at position 126. Among these three GNMTs, there are 31 differences in the amino acid residues. Most of these are conservative substitutions, but there are also substitutions that could potentially change the local conformation of the proteins, such as a $\mathrm{K}$ to $\mathrm{H}$ and $\mathrm{A}$ to $\mathrm{S}$ in the region $113-119$, or the $\mathrm{D}$ to $\mathrm{G}$ substitution at the C-terminus. The crystal structure of rat GNMT indicates that the differences in amino acid sequences are located on the surface of the protein molecule, far from the active site. Hence, they should not directly affect the catalysis. However, differences in amino acid composition result in differences in general protein characteristics. For instance, the predicted value of $\mathrm{pI}$ for human GNMT is 6.6, while for the mouse and rat forms it is 7.1.

\section{Quaternary Structure}

Expressed human, rat and mouse GNMTs were tetramers as they were eluted from the Superose-12 column in one peak with elution volumes of $12.55,12.73$ and 12.90 $\mathrm{mL}$ for human, mouse and rat proteins respectively. This corresponds to an apparent $M_{r}$ of $130 \mathrm{kDa}$ (data not shown). While the differences in elution volume of GNMTs were small, they were reproducible in different buffers. This could be explained as a result of the smaller Stokes radii of mouse and rat enzymes compared to human protein and is a result of a more compact conformation of rat and mouse GNMTs.

\section{Enzyme Kinetics}

The kinetic parameters for human, rat and mouse GNMTs are presented in Table II. The $K_{m}(\mathrm{SAM})$ values for the human enzyme are higher than the corresponding values for mouse and rat. The $K_{m}$ (gly) value of human GNMT is three times that of the rat enzyme. The human enzyme has the highest $k_{\text {cat }}$, while the rat has the lowest. 
This was measured when the enzyme was saturated with both substrates and is effectively the turnover number. When the $k_{\text {cat }} / K_{m}$ is calculated for the individual substrates, the value for SAM of the human enzyme remains highest, but the opposite is the case with respect to glycine. This latter value is an index of the efficiency of the enzyme. The human enzyme appears to be most efficient with regard to SAM but not to glycine.

A
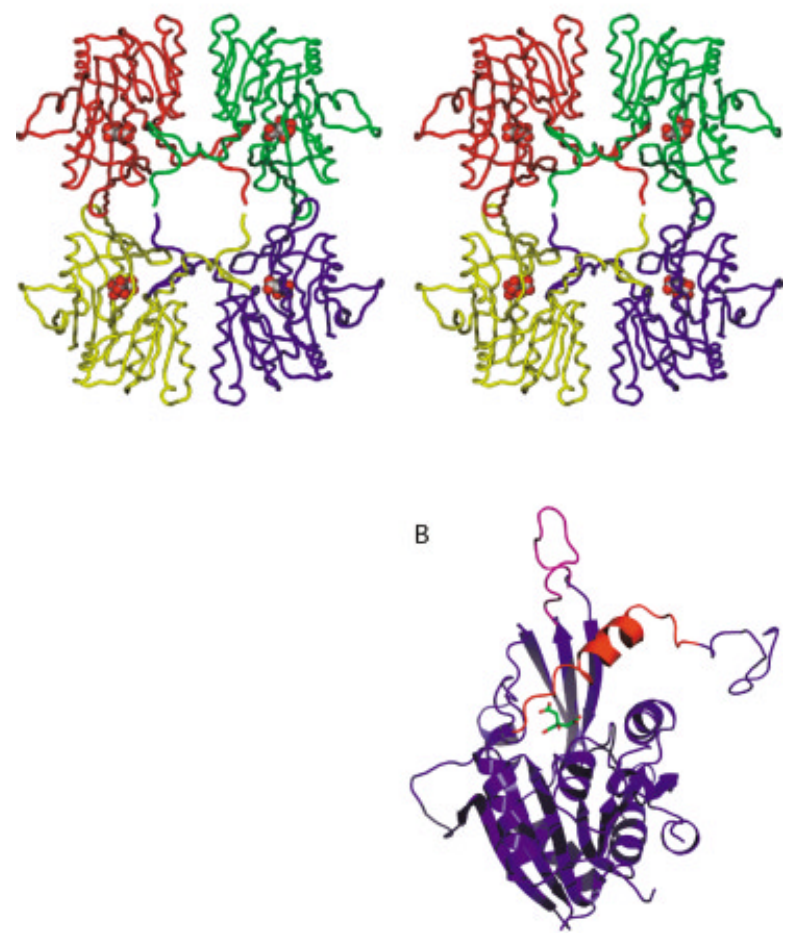

Fig. 2. (a) A ribbon representation (stereo) of the human GNMT tetramer. Protein monomers are shown in different colors; the active site-bound citrate molecules are included as space-filled renderings. (b) Structure of a monomer of human GNMT with bound citrate molecule in the active site. Significantly disordered parts of the monomer: a flexible loop 225-236 (magenta) and a helical region 22-41 (red) are shown.
Previous studies comparing GNMT from various species showed that human GNMT had a higher $[S]_{0.5}$ for SAM and glycine than did rat GNMT. ${ }^{3}$ It should be noted that these previous studies were done with crude ammonium sulfate fractions from liver, and the results reported here are for the purified recombinant enzymes expressed in $E$. coli. Moreover, rat liver GNMT is phosphorylated, while the recombinant enzymes expressed in $E$. coli probably are not. ${ }^{27}$ It is not known what effect this post-translational modification has on the kinetics of GNMT. Therefore it is probably not informative to try to compare the relative activities from different species when there is such a difference in the protein preparations. In the case of rat GNMT, we have measured the $[S]_{0.5}$ for SAM and glycine in both purified liver ${ }^{28}$ and recombinant enzymes (Table II). For rat liver, the $[S]_{0.5}$ for SAM and glycine were 0.39 $\mathrm{m} M$ and $0.91 \mathrm{~m} M$, respectively. For the rat recombinant enzyme, the corresponding values were $0.22 \mathrm{mM}$ and 12.2 $\mathrm{m} M$, respectively. We suggest that the post-translational modification affects the affinities of the substrates.

\section{Conformations of Human and Mouse GNMTs}

The crystal structure analyses of human GNMT and two crystal forms of mouse GNMT, as expected, revealed very similar molecular conformations as well as the typical conformation for rat GNMT tetrameric assembly [Fig. 2 (a)]. The highest root mean square (RMS) deviation of $0.727 \AA$ (calculated over 274 equivalent CA atoms) was observed between the monomers of the human and rat enzymes. The overall molecular conformation is basically the previously described 'closed' form of rat GNMT. However, significant conformational differences were observed in the 225-233 loops (the region that, in the rat enzyme, is disordered in the 'open' and 'active' forms of the enzyme). In the human GNMT structure, as well as in the structures of the mouse enzyme, loops 225-235 are highly disordered. These new structures display features of the 'inactive' and 'active' forms of the rat enzyme. Loops 127-134, which contain the two amino acid insertion in human GNMT, are also disordered, just as in the 'active' form of rat GNMT. In contrast, this loop is ordered in both
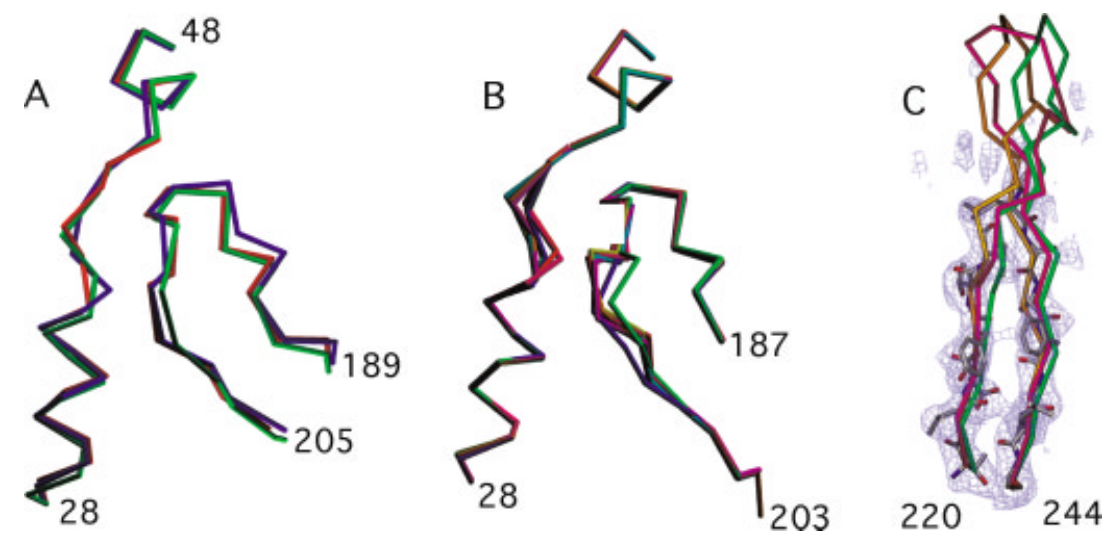

Fig. 4. Flexible elements in GNMT structures. (a) Superimposition of residues 28-48 and 189-205 from eight independent monomers in monoclinic form of mouse GNMT. (b) Superimposition of the same parts in rat (shown in green), mouse (red) and human (green) GNMT. (c) Representative portion of the final $2 F_{o}-F_{c}$ map calculated around the 'missing' loop 225-236 in the structure of human GNMT contoured at $1 \sigma$. Superimposed equivalent loops from inactive (orange), open (green) and active (magenta) forms of rat GNMT are shown. 


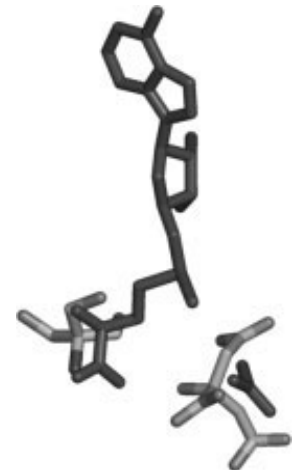

Fig. 3. Superimposition of the active site in the active form of rat GNMT (shown in dark gray) on the active site bound citrate molecule in human GNMT and the Tris molecule in tetragonal form of mouse GNMT.

forms of mouse GNMT, and so in this area the mouse enzyme resembles the inactive conformation of rat GNMT. Another significantly flexible part of the human GNMT molecule includes $\mathrm{N}$-terminal domain residues $21-41$, the region that 'corks' the active site in the closed form of rat GNMT [Fig. 2(b)]. In addition, residues 1-4 are not visible on electron density maps. The breakage of the antiparallel $\beta$-sheet hydrogen bonds between the tail sections of the $\mathrm{N}$-termini (residues 2-7) of adjacent subunits is proposed to be the first of a series of conformational changes that must occur in the catalytic cycle of the rat enzyme. ${ }^{12}$ In this model, SAM competes with the U loop (residues 9-20) for the active site. Depending on the concentration of SAM, the active open (high SAM concentration) or the inactive closed (low SAM concentration) conformation is formed. In the 'open' form, residues 21-40 have turned into a solvent region, and thus glycine can enter the active site through the channel opened by the movement of helical residues 24-55 (helix $\mathrm{a}^{\prime}$ as designated by $\mathrm{Fu}$ at al. ${ }^{10}$ ). Human GNMT was crystallized without the addition of SAM, and thus the SAM binding site was filled by the U loop. The bound citrate molecule, however, was found in the glycine binding site in the position of the acetate ion in the active form of rat GNMT (Fig. 3) with similar hydrogen bonds to Y33, Y222 (Y220 in rat) and N140 (N138 in rat). Hence, a disordered helix a' could represent a likely entrance for a substrate into the active site in human GNMT. Superimposition of molecules of available crystal structures of GNMTs has shown that residues 37-42 of helix a' are indeed between the most flexible parts in GNMT molecules [Fig. 4(a)]. They even have different conformations in all eight independent protein monomers in the monoclinic form of mouse GNMT [Fig. 4(b)].

It is interesting that Tris molecules bind in a different place in the tetragonal form of mouse GNMT compared to the location of citrate binding at the glycine site in human GNMT (Fig. 3). A comparison to the structure of the active form of rat GNMT shows that Tris occupies the SAM binding site. The active site is completely empty in the monoclinic form of mouse GNMT. Residues 225-236 are still highly disordered, however, just as they are in the tetragonal form and the structure of human GNMT.

Of all known methyltransferases, GNMT is unique in that it has an additional S-domain (residues 176-242).
Hence, it is reasonable to assume that this domain should be involved in the glycine-N-methyltransferase reaction. Indeed, the S-domain covers the active site and represents a very mobile part of the molecule. Residues 188-192 are flexible in all known GNMT structures except for the apo-form of rat GNMT. For example, this region of the polypeptide chain has two conformations in the inactive form of rat GNMT. In addition, the eight monomers of the mouse monoclinic structure display different conformations in this region [Fig. 4(b)]. The 'apex' of the S-domain, the loop composed of residues 220-244, has distinctly different conformations in the inactive, active and open forms of rat GNMT [Fig. 4(c)]. This part of the structure is significantly shifted in the open form compared to the inactive one. It has a completely different conformation in the active form of rat GNMT, with residues 226-234 being significantly disordered (their B-factors are higher than 50 $\AA^{2}$ ). In the human and mouse GNMT structures, the electron density is completely missing for those residues, suggesting that they are highly mobile or disordered [Fig. 4(c)].

All these observations show that, despite the fact that overall molecular structures of human and mouse GNMTs resemble the one observed in the inactive form of rat GNMT, the human and mouse GNMT enzymes are more flexible than the rat enzyme. The observed high disorder of loop 225-233 in the human and mouse GNMTs and loop 127-134 in human GNMT makes these regions resemble the active conformation of rat GNMT. The observed conformational heterogeneity of residues $37-42$ and 188-192, positions close to the active site, in the human and mouse GNMTs, along with the appearance of buffer molecules in the active sites of the proteins (binding of a citrate molecule in the glycine binding site of human GNMT), also suggests that the observed conformations of human and mouse GNMTs may be considered to be equivalent to the intermediate states between the inactive and the active forms of the rat enzyme.

\section{CONCLUSIONS}

The results of crystal structure analysis have shown that both human GNMT and mouse GNMT in both crystal forms are more flexible than the rat enzyme. This is in agreement with the catalytic efficiency of the rat, mouse and human recombinant glycine $\mathrm{N}$-methyltransferases.

\section{ACKNOWLEDGEMENTS}

We would like to acknowledge support from NIH grants DK15289 and DJ54859 and from the office of Veterans Affairs to C.W. and from NIH GM55420 and the Louisiana Governor's Biotechnology Initiative to M.E.N.

\section{REFERENCES}

1. Ogawa H, Fujioka M. Purification and properties of glycine N-methyltransferase from rat liver. J Biol Chem 1982;257:34473452 .

2. Heady JE, Kerr SJ. Purification and characterization of glycine N-methyltransferase. J Biol Chem 1973;248:69-72.

3. Ogawa H, Gomi T, Fujioka M. Mammalian glycine N-methyltransferases. Comparative kinetic and structural properties of the enzymes from human, rat, rabbit and pig livers. Comp Biochem \& Physiol B: Comp Biochem 1993;106B:601-611. 
4. Cantoni GL, Richards HH, Chiang PK. Inhibitors of S-adenosylhomocysteine hydrolase and their role in the regulation of biological methylation. In Usdin E, Borchardt RT, Creveling CR, editors. Transmethylation. New York: Elsevier; 1978, p 155-64.

5. Suzuki N, Wagner C. Purification and characterization of a folate binding protein from rat liver cytosol. Arch Biochem Biophys 1980;199:236-248.

6. Wagner C, Briggs WT, Cook RJ. Inhibition of glycine Nmethyltransferase activity by folate derivatives: implications for regulation of methyl group metabolism. Biochem \& Biophys Res Comm 1985; 127:746-752.

7. Balaghi M, Horne DW, Wagner C. Hepatic one-carbon metabolism in early folate deficiency in rats. Biochem J 1993;291:145-149.

8. Yeo EJ, Wagner C. Tissue distribution of glycine N-methyltransferase, a major folate-binding protein of liver. Proc Natl Acad Sci USA 1994;91:210-214.

9. Wagner C, Decha-Umphai W, Corbin J. Phosphorylation modulates the activity of glycine N-methyltransferase, a folate binding protein. In vitro phosphorylation is inhibited by the natural folate ligand. J Biol Chem 1989;264:9638-9642.

10. Fu Z, Hu Y, Konishi K, Takata Y, Ogawa H, Gomi T, Fujioka M, Takusagawa F. Crystal structure of glycine N-methyltransferase from rat liver. Biochem 1996;35:11985-11993.

11. Pattanayek R, Newcomer ME, Wagner C. Crystal structure of apo-glycine N-methyltransferase (GNMT). Protein Sci 1998;7: $1326-1331$.

12. Huang Y, Komoto J, Konishi K, Takata Y, Ogawa H, Gomi T, Fujioka M, Takusagawa F. Mechanisms for auto-inhibition and forced product release in glycine N-methyltransferase: crystal structures of wild-type, mutant R175K and S-adenosylhomocysteine-bound R175K enzymes. J Mol Biol 2000;298:149-162.

13. Takata Y, Huang Y, Komoto J, Yamada T, Konishi K, Ogawa H, Gomi T, Fujioka M, Takusagawa F. Catalytic mechanism of glycine N-methyltransferase. Biochem 2003;42:8394-8402.

14. Ogawa H, Gomi T, Takusagawa F, Fujioka M. Structure, function and physiological role of glycine N-methyltransferase. Int $\mathrm{J}$ Biochem Cell Biol 1998;30:13-26.

15. Mudd SH, Cerone R, Schiaffino MC, Fantasia AR, Minniti G, Caruso U, Lorini R, Watkins D, Matiaszuk N, Rosenblatt DS, Schwahn B, Rozen R, LeGros L, Kotb M, Capdevila A, Luka Z, Finkelstein JD, Tangerman A, Stabler SP, Allen RH, Wagner C. Glycine N-methyltransferase deficiency: a novel inborn error causing persistent isolated hypermethioninaemia. J Inherit Metab Dis 2001;24:448-464.
16. Luka Z, Cerone R, Phillips J, Mudd HS, Wagner C. Mutations in human glycine $\mathrm{N}$-methyltransferase give insights into its role in methionine metabolism. Hum Genet 2002;110:68-74.

17. Augoustides-Savvopoulou P, Luka Z, Karyda S, Stabler SP, Allen RH, Patsiaoura K, Wagner C, Mudd SH. Glycine N-methyltransferase deficiency: a new patient with a novel mutation. J Inherit Metab Dis 2003;26:745-759.

18. Luka Z, Wagner C. Effect of naturally occurring mutations in human glycine N-methyltransferase on activity and conformation. Biochem Biophys Res Commun 2003;312:1067-1072.

19. Luka Z, Wagner C. Expression and purification of glycine Nmethyltransferases in Escherichia coli. Protein Expr Purif 2003;20: $280-286$.

20. Otwinowski Z, Minor W. Processing of X-ray diffraction data collected in oscillation mode. Methods Enzymol A 1997;276:307326.

21. Rossmann MG, van Beek CG. Data processing. Acta Crystallogr D Biol Crystallogr 1999;55:1631-1640.

22. The CCP4 suite: programs for protein crystallography. Acta Crystallogr D Biol Crystallogr 1994;50:760-763.

23. Adams PD, Pannu NS, Read RJ, Brunger AT. Cross-validated maximum likelihood enhances crystallographic simulated annealing refinement. Proc Natl Acad Sci USA 1997;94:5018-5023.

24. Jones TA, Zou JY, Cowan SW, Kjeldgaard M. Improved methods for building protein models in electron density maps and the location of errors in these models. Acta Crystallogr A 1991;4:110 119.

25. Cook RJ, Misono KS, Wagner C: Identification of the covalently bound flavin of dimethylglycine dehydrogenase and sarcosine dehydrogenase from rat liver mitochondria. J Biol Chem 1984;259: 12475-12480.

26. Smith PK, Krohn RI, Hermanson GT, Mallia AK, Gartner FH, Provenzano MD, Fujimoto EK, Goeke NM, Olson BJ, Klenk DC. Measurement of protein using bicinchoninic acid. Anal Biochem 1985;150:76-85.

27. Lucher LA, Loewenstein PM, Green M. Phosphorylation in vitro of Escherichia coli-produced 235R and 266R tumor antigens encoded by human adenovirus type 12 early transformation region $1 \mathrm{~A}$ J Virol 1985;56:183-193.

28. Yeo EJ, Briggs WT, Wagner C. Inhibition of glycine N-methyltransferase by 5 -methyltetrahydrofolate pentaglutamate. J Biol Chem 1999;274:37559-37564. 